\title{
Comparative Study of Marketed and Novel Colloidal Formulation for Topical Delivery of 5-Fluorouracil to Skin Cancer Cells: Ex-Vivo Release Study and Cytotoxicity Analysis
}

\author{
(1) Harish SHARMA, ${ }^{1,2}$ (1) Gyanesh Kumar SAHU, ${ }^{3,4}$ (1) Swarnali Das PAUL, ${ }^{3}$ (D) Chanchal Deep KAUR ${ }^{1}$ \\ 'Department of Pharmaceutics, Shri Rawatpura Sarkar Institute of Pharmacy, Chhattisgarh-India \\ 2Department of Pharmaceutics, Rungta Institute of Pharmaceutical Sciences, Chhattisgarh-India \\ ${ }^{3}$ Department of Pharmaceutics, Faculty of Pharmaceutical Science, Shri Shankaracharya Group of Institute, Chhattisgarh-India \\ ${ }^{4}$ Department of Pharmaceutics, Rungta College of Pharmaceutical Sciences and Research, Chhattisgarh-India
}

\begin{abstract}
OBJECTIVE
The objective of the present work was to compare the efficacy of two formulations (microemulsion [ME] and nanoemulsion [NE]) with marketed product on skin cancer cell lines.

\section{METHODS}

For this purpose, we studied two parameters, that is, drug release both in ex vivo conditions and cytotoxicity profile. For ex vivo skin permeability studies skin of male Sprague Dawley rats, goats, and human cadaver was selected. For in vitro cytotoxicity study, SK-MEL-5 melanoma cell lines were used. \% cell death was concentration dependent.
\end{abstract}

\section{RESULTS}

The highest concentration $(96 \mu \mathrm{M})$ maximum cell death was found. The nanoemusion had highest inhibitory effect in any concentration than control or ME. Similar results were obtained for permeation study. NE had better permeation property than other.

\section{CONCLUSION}

Based on the permeation results, we found that highest amount of 5-fluorouracil collected in the viable part of the human cadaver and goat skin model from NE as compared to other preparations. Finally, it can be concluded that NE of the model drug had better properties in cytotoxicity and skin permeation than marketed topical product.

Keywords: 5-Fluorouracil; microemusion; nanoemulsion; skin cancer.

Copyright @ 2022, Turkish Society for Radiation Oncology

\section{Introduction}

Dermal delivery of drug is the most popular route for the management of skin cancers topically. The method of drug delivery is not as invasive as compared to par- enteral. In addition, we can reduce systemic toxins associated with cancer drugs.[1]

5-Fluorouracil (5-FU) is used as an anti-cancer drug in the treatment of various diseases, both localized, such as skin cancer and actinic keratosis.[2] How- 
ever, the main drawback of this drug is low permeability and solubility from cream, ointment, and gels to attain therapeutic concentration.[1]

The existing formulas are available for the topical application of 5-FU, in the form of a cream or solution, and the concentration of $5-\mathrm{FU}$ is set up to $0.50 \%$, which can be taken once a day, for topical use. The commercially available drugs have the disadvantage of a very short retention time and less permeation $[3,4]$ results such as irritation of the skin, dryness, swelling, burning and pain. This kind of reaction can be extended for a further 2 weeks. [5] Therefore, a novel formulation is required to avoid this type of side effects and need to enhance penetration into the skin.[6]

Management of tumorigenic effects on the skin, as well as minimizing local and systemic side effects of 5-FU should penetrate the skin layers and exert effects on the epidermis and dermis. The existing routes of administration of 5-FU, have been placed on intravenous and local routes of administration; $[7,8]$ this study was focused on development of 5-FU-novel colloidal topical formulation to skin cancer and compared with conventional marketed preparation.

\section{Materials and Methods}

5-FU gift samples from Neon laboratories Mumbai, India, rutin, tween 80 (Molychem, Mumbai, INDIA), isopropyl alcohol (molychem, Mumbai), oleic acid (Central Drug House Private Ltd., New Delhi, India), and dimethyl sulfoxide (Sisco research Laboratory, Mumbai, India), ethanol, n-butanol, propylene glycol, polyethylene glycol, span 60 , span 80 , isopropyl mystriate, and triple distilled water were used during the experiment.

To produce 5-FU microemulsions (MEs) and nanoemulsion (NE), surfactant-cosurfactant was mixed with oil. Distilled water was added drop wise with the help of a burette by gentle stirring at a constant temperature. The surfactant and co-surfactant were mixed into three particular ratios 1:1, 2:1, and 3:1.

\section{Characterization}

\section{Ex vivo skin permeation studies}

The skin of male Sprague Dawley (SD) rats, goats, and human cadaver was selected for ex vivo skin permeability studies. Male SD rats, 6-7 weeks of age and weighing about 180-200 g. The Institutional Committee for Animal Ethics (approval no. SRIP/IAEC/2019-20/191/12) was approved for experimental protocol. The rats were obtained from research institute's animal house. Goat-skin, weighing 8-10 g taken from a local slaughter house in Bhilai, Chhattisgarh, India, as well as the human skin, which obtained from the Shri Shankarcharyacharya Institute of Medical Sciences (letter no. SSIMS/320).

\section{Permeation studies}

The ex vivo skin penetration tests were performed to determine and compare skin penetration parameters between 5FU loaded ME, NE, and marketed cream. The experiments were carried out in the static Franz diffusion cell ( $9 \mathrm{~mm}$ clear glass jacket). The Franz diffusion cell consisting of two compartments: The receptor and donor compartment.

To preceding permeation study, isolated part of back skin of human cadaver, goat, and rat was mounted between the receptor and the donor compartments, while keeping the human cadaver, goat, and rat stratum corneum site of the skin facing toward the donor compartment.

At certain time intervals 1, 2, 4, 6, 8, and 12, after 24 h, $0.5 \mathrm{ml}$ of the volume was taken from the intake opening and immediately replaced with an equal volume of fresh medium at the same temperature, to maintain the sum of the effects of phase at a constant level. The value of 5-FU in the collected samples was measured using the HPLC method.

5-FU permeability was determined in the different skin types human cadaver, rat and goat, for each of the 5-FU cream, 5-FU ME, and 5-FU NEs with 5-FU control, For calculating permeability coefficient (Kp), steady state for human cadaver, rat, and goat skin divided with initially concentration ( $\mathrm{C} 0$ ) of 5-FU. A graph is plotted of the period of time between the copenetration of 5 -FU (in $\mu \mathrm{g} / \mathrm{cm} 2$ ) and time (hours) to have a specific part of the diffusion cell area.

Enhancement ratio and permeability coefficient were determined by use of equation 1 and 2.[9]

$\mathrm{Kp}=\frac{\mathrm{Css}}{\mathrm{C}_{\mathrm{o}}}$

$\mathrm{Er}=\frac{\text { Css of formulation }}{\text { Css of control }}$

\section{Cytotoxicity studies}

For in vitro cytotoxicity study SK-MEL-5 melanoma cell lines were used, with the relative effects of selected formulations and control. MTT measures cellular respiration, and the amount of synthetic formazan in the culture correlates to the number of live cells. A change in cell number corresponds to a change in the amount 
of formazan generated, which measures the degree of cytotoxicity induced by the medication. A drug's IC50 (concentration) has the capacity to kill $50 \%$ of cells and can calculate the degree of its cytotoxic effect.

\section{Results}

\section{Ex vivo Data of Different 5-FU Formulation}

Ex vivo permeation studies of 5-FU loaded different formulation on the skin of human cadaver, rats, and goats are shown on Figure 1. The permeation of 5-FU from NE for rat skin model was greater than human cadaver and goat skin. There was no significant skin permeation difference between human cadaver and goat skin model. However, in rat and human cadaver skin statistically noteworthy difference was found.

Table 1 shows the Enhancement Ratio (EnR), the steady-state flux (Css), and the Permeability coefficient (Kp). Figure 2 depicts all results relating to $5-\mathrm{FU} \%$ penetration, accumulation in the SC, and viable skin. In place of retention in the skin, 5-FU is highly permeable for 5-FU-NE in the human cadaver skin model. After $24.0 \mathrm{~h}$, the retention of 5-FU in the SC and viable section of the different skins was assessed. According to the permeation results, 5-FU-NE collected the most 5 -FU in the viable part of the human cadaver and goat skin model as compared to the other preparations, 5-FU-C and 5-FU-ME for the rat skin model.

\section{In vitro Cell Lines Studies}

The in vitro therapeutic efficacy of optimized 5-FU-ME and 5-FU-NE on melanoma cancer cell lines has been evaluated (SK-MEL-5 type). The melanoma cell lines were tested in vitro for cytotoxicity (percent) using various molar concentrations of 5-FU from 5-FU-C, 5-FU-ME, and 5-FU-NE. Cell survival (percent) was also assessed using different molar concentrations of 5-FU $(3,6,12,24,48$, and $98 \mathrm{M})$ in 5-FU-C, 5-FU$\mathrm{ME}$, and 5-FU-NE (Fig. 3). On melanoma cell lines, free 5-FU demonstrated no significant cell suppression (Fig. 4). Table 2 shows the cytotoxicity percentage. Percent cell death was concentration dependent. In the highest concentration $(96 \mu \mathrm{M})$, maximum cell death was found. For ME, $65 \%$ death was obtained and $82 \%$ inhibition was found in case of NE. The nanoemusion had highest inhibitory effect in any concentration than control or ME. This phenomenon may be obtained due to greater cell penetration properties by NE.

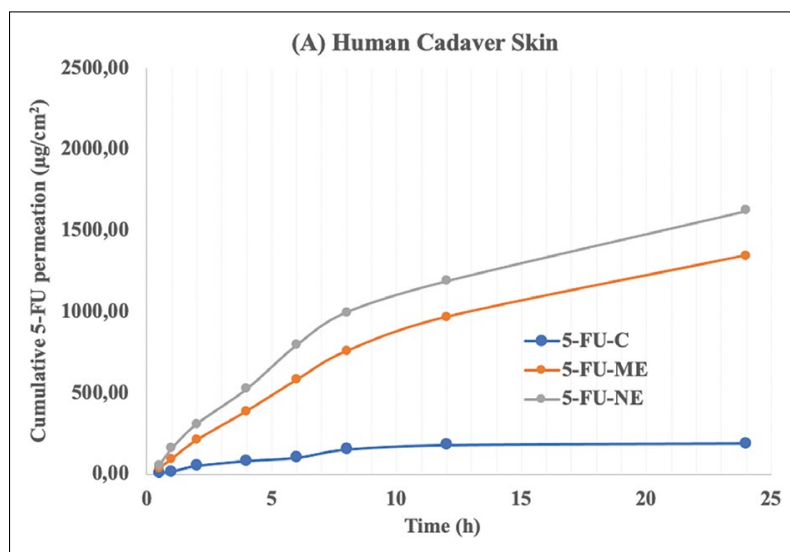

Fig. 1. Ex vivo skin permeation studies of 5-fluorouracil from control, microemulsion, and nanoemulsion formulations on human cadaver skin.
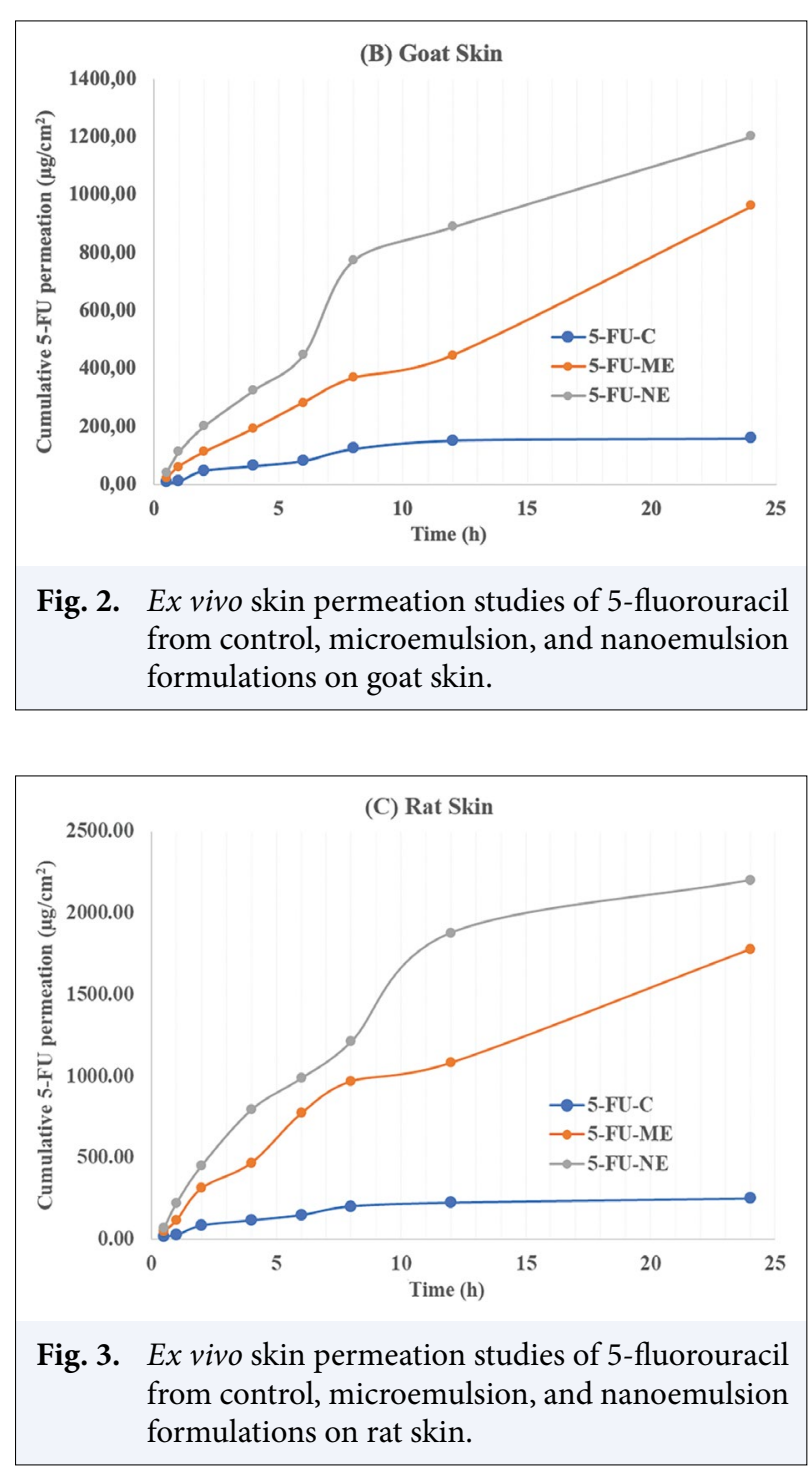

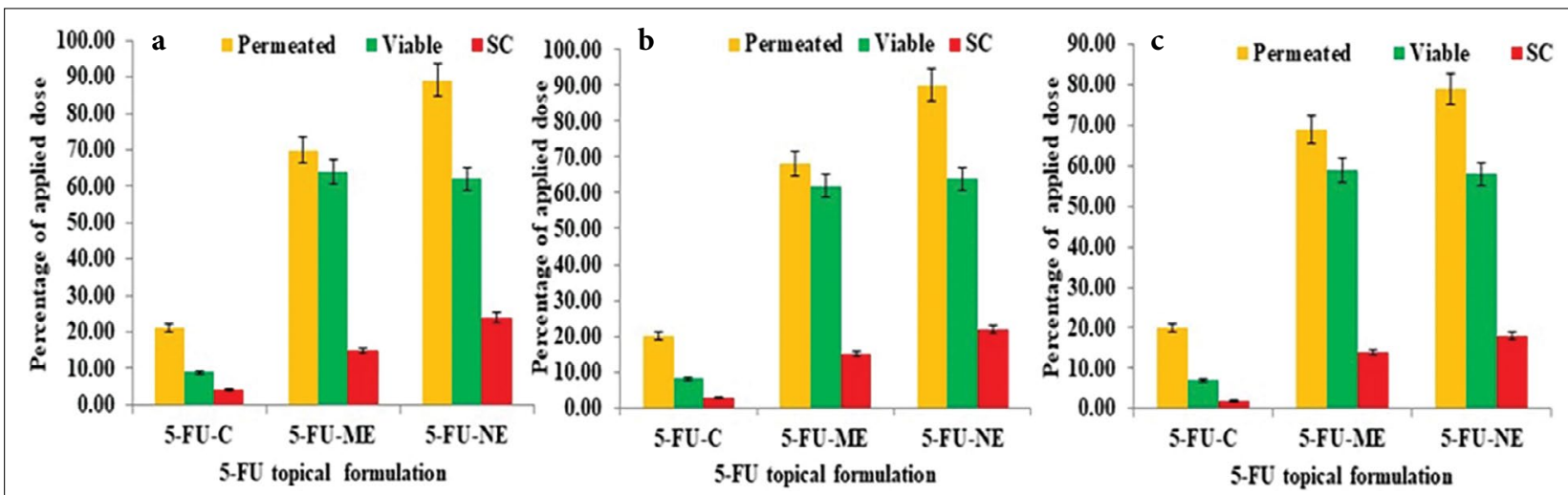

Fig. 4. Ex vivo 5-fluorouracil (5-FU) permeability and skin retention in the SC and viable section of the skin [(a) Human cadaver skin, (b) Goat skin, (c)] Rat skin from the 5-FU-Control, 5-FU-microemulsion, and 5-FU-nanoemulsion formulations.

5-FU: 5-Fluorouracil; ME: Microemulsions; NE: Nanoemulsion; SC: Stratum corneum.

\section{Discussion}

Different nano drug delivery systems include microemulsions and nanoemulsions have been investigated for superior topical application since years as they offer many advantages including successful preparation of hydrophobic molecules by enhancing their permeability, solubility and therefore bioavailability. This approach has been lead for using hydrophobic molecules to a variety of routes of administration, including topical application. The present study was aimed to formulate an anticancer agent into microemulsions and nanoemulsions and compare its efficacy with existing conventional dosage forms. As discussed earlier the permeation results showed, 5-FU-NE collected the most 5-FU in the viable part of the human cadaver

Table 1 Permeability 5-FU formulation on various animal skins (human cadaver, goat, and rat)

\begin{tabular}{|c|c|c|c|c|c|c|c|c|c|}
\hline \multirow[t]{2}{*}{ Skin model } & \multicolumn{3}{|c|}{$\begin{array}{l}\text { Steady-state flux } \\
\text { (Css) }\left(\mu / \mathrm{cm}^{2} / \mathrm{h}\right)\end{array}$} & \multicolumn{3}{|c|}{$\begin{array}{l}\text { Permeability coefficient } \\
(\mathrm{Kp})\left(\mu \mathrm{g} \times 10^{-2}\right)\end{array}$} & \multicolumn{3}{|c|}{$\begin{array}{l}\text { Enhancement } \\
\text { ratio (EnR) }\end{array}$} \\
\hline & Control & NE & ME & Control & NE & ME & Control & NE & ME \\
\hline Human cadaver skin & $0.949 \pm 0.39$ & $8.166 \pm 0.60$ & $9.846 \pm 0.81$ & $0.089 \pm 0.002$ & $0.814 \pm 0.012$ & $0.978 \pm 0.014$ & - & 8.50 & 10.26 \\
\hline Goat skin & $0.946 \pm 0.38$ & $8.203 \pm 0.61$ & $9.897 \pm 0.86$ & $0.091 \pm 0.002$ & $0.819 \pm 0.011$ & $0.985 \pm 0.016$ & - & 8.56 & 10.30 \\
\hline Rat skin & $1.270 \pm 0.53$ & $10.337 \pm 0.93$ & $12.018 \pm 1.14$ & $0.134 \pm 0.004$ & $1.032 \pm 0.059$ & $1.201 \pm 0.072$ & - & 7.64 & 8.89 \\
\hline
\end{tabular}

5-FU: 5-Fluorouracil; Css: Steady-state flux; Kp: Permeability coefficient; EnR: Enhancement ratio; ME: Microemulsions; NE: Nanoemulsion

Table 2 5-FU-C, 5-FU-ME, and 5-FU-NE cytotoxicity on melanoma cancer cell lines $(\mathrm{n}=3)$

\begin{tabular}{lccc} 
Concentration $(\boldsymbol{\mu M})$ & \multicolumn{3}{c}{ Percentage cell death } \\
\cline { 2 - 4 } & $\mathbf{5 - F U - C}$ & $\mathbf{5 - F U - M E}$ & 0 \\
\hline 0 & 0 & 0 & $5.59 \pm 0.77$ \\
3 & $-16.23 \pm 0.64$ & $3.44 \pm 0.82$ & $15.67 \pm 1.29$ \\
6 & $-12.89 \pm 0.66$ & $9.98 \pm 0.99$ & $38.41 \pm 2.26$ \\
12 & $-1.13 \pm 0.11$ & $31.84 \pm 1.02$ & $52.22 \pm 2.29$ \\
24 & $2.01 \pm 0.17$ & $36.26 \pm 2.22$ & $61.11 \pm 3.39$ \\
48 & $3.03 \pm 0.82$ & $54.36 \pm 2.88$ & $81.13 \pm 4.44$ \\
96 & $3.99 \pm 0.54$ & $64.79 \pm 3.39$ & 5 \\
\hline
\end{tabular}


and goat skin model as compared to the other preparations, 5-FU-C and 5-FU-ME for the rat skin model. In cytotoxicity study ME offered $65 \%$ cell death and NE caused $82 \%$ inhibition. Further it also noted that NE had highest inhibitory effect in any concentration than control or ME. All these results clearly pointed the better efficacy of NE in delivering the drug than other preparations via topical route.

\section{Conclusion}

Colloidal delivery systems such as NEs or MEs have found increased applications nowadays to encapsulate and deliver different bioactive compounds. The small globule size of these systems $(\mathrm{r}<100 \mathrm{~nm})$ has a number of potential benefits. However, a ME is thermodynamically stable and a NE is not. In this study we found NE had better cytotoxic and permeation properties than other formulations. This may be attributed by the greater penetration properties of NE due to smaller size range than others. Finally, it can be concluded that $\mathrm{NE}$ of this model drug was more effective than ME or marketed topical product.

Acknowledgments: The authors would like to express thanks Neon Laboratories Ltd., Mumbai, India, for providing the drug as a gift sample. We also express our kind gratitude to Indian Institute of Integrative Medicine, Jammu, India and All India Medical Institute of Medical Sciences, New Delhi, India.

Peer-review: Externally peer-reviewed.

Conflict of Interest: All authors declared no conflict of interest.

Ethics Committee Approval: The study was approved by The Shri Rawatpura Sarkar Institute of Pharmacy Ethics Committee (No: SRIP/IAEC/2019-20/191/12, Date: 22/02/2020).

Financial Support: This study has received no financial support.

Authorship contributions: Concept - H.S.; Design - H.S.; Supervision - C.D.K.; Funding - C.D.K.; Materials - S.D.P.;
Data collection and/or processing - G.K.S.; Data analysis and/or interpretation - H.S.; Literature search - G.K.S.; Writing - S.D.P.; Critical review - H.S.

\section{References}

1. Rajinikanth PS, Chellian J. Development and evaluation of nanostructured lipid carrier-based hydrogel for topical delivery of 5-fluorouracil. Int J Nanomedicine 2016;11:5067-77.

2. Van Ruth S, Jansman FGA, Sanders CJ. Total body topical 5-fluorouracil for extensive non-melanoma skin cancer. Pharm World Sci 2006;28(3):159-62.

3. Saif MW, Choma A, Salamone SJ, Chu E. Pharmacokinetically guided dose adjustment of 5-fluorouracil: a rational approach to improving therapeutic outcomes. J Natl Cancer Inst 2009;101(22):1543-52.

4. Khandavilli S, Panchagnula R. Nanoemulsions as versatile formulations for paclitaxel delivery: Peroral and dermal delivery studies in rats. J Invest Dermatol. 2007;127(1):154-62.

5. Elmeshad AN, Tadros MI. Transdermal delivery of an anti-cancer drug via W/O emulsions based on alkyl polyglycosides and lecithin: Design, characterization, and in vivo evaluation of the possible irritation potential in rats. AAPS PharmSciTech. 2011;12(1):1-9.

6. Liu F, Xiao YY, Ping QN, Yang C. Water in oil microemulsions for transdermal delivery of fluorouracil. Yaoxue Xuebao 2009;44(5):540-7.

7. Chinembiri TN, Gerber M, du Plessis L, du Preez J, du Plessis J. Topical delivery of 5-fluorouracil from PheroidTM formulations and the in vitro efficacy against human melanoma. AAPS PharmSciTech 2015;16(6):1390-9.

8. Patzelt A, Antoniou C, Sterry W, Lademann J. Skin penetration from the inside to the outside: A review. Drug Discov Today Dis Mech 2008;5(2):229-35.

9. Thakur R, Anwer MK, Shams MS, Ali A, Khar RK, Shakeel F, Taha EI. Proniosomal transdermal therapeutic system of losartan potassium: Development and pharmacokinetic evaluation. J Drug Target 2009;17(6):442-9. 